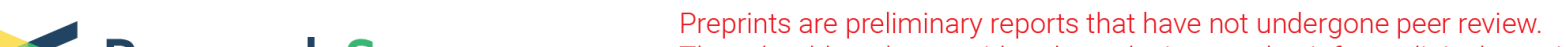 $\begin{array}{ll}\text { Research Square } & \begin{array}{l}\text { They should not be considered conclusive, used to inform clinical practice, } \\ \text { or referenced by the media as validated information. }\end{array}\end{array}$
}

\section{The SARS-CoV-2 B.1.1.529 Omicron virus causes attenuated infection and disease in mice and hamsters}

\section{Michael Diamond ( $\sim$ mdiamond@wustl.edu )}

Washington University in Saint Louis https://orcid.org/0000-0002-8791-3165

\section{Peter Halfmann}

Influenza Research Institute, Department of Pathobiological Sciences, School of Veterinary Medicine, University of Wisconsin, Madison, WI, USA

\section{Tadashi Maemura}

University of Wisconsin

\section{Kiyoko Iwatsuki-Horimoto}

University of Tokyo https://orcid.org/0000-0002-8266-020X

\section{Shun lida}

Department of Pathology, National Institute of Infectious Diseases, Tokyo

\section{Maki Kiso}

Institute of Medical Sciences, University of Tokyo

\section{Suzanne Scheaffer}

Washington University in St. Louis

\section{Tamarand Darling}

Washington University School of Medicine

\section{Astha Joshi}

Washington University School of Medicine https://orcid.org/0000-0003-4914-8228

\section{Samantha Loeber}

University of Wisconsin-Madison

\section{Stephanie Foster}

Center for Childhood Infections and Vaccines of Children's Healthcare of Atlanta, Department of

Pediatrics, Emory Vaccine Center, Emory University School of Medicine

\section{Baoling Ying}

Washington University School of Medicine

\section{Bradley Whitener}

Departments of Medicine, Washington University School of Medicine, St. Louis, MO, USA https://orcid.org/0000-0001-6652-0701

\section{Katharine Floyd}

Emory University School of Medicine https://orcid.org/0000-0002-0866-6961 


\section{Michiko Ujie}

Division of Virology, Institute of Medical Science, University of Tokyo

\section{Noriko Nakajima}

National Institute of Infectious Diseases

\section{Mutsumi Ito}

University of Tokyo, Institute of Medical Science

\section{Ryan Wright}

Influenza Research Institute, Department of Pathobiological Sciences, School of Veterinary Medicine, University of Wisconsin-Madison

\section{Ryuta Uraki}

National Center for Global Health and Medicine Research Institute https://orcid.org/0000-0003-08901922

\section{Rong Li}

Department of Animal Dairy, and Veterinary Sciences, College of Agriculture and Applied Sciences, Utah State University

\section{Yuko Sakai}

Institute of Medical Sciences, University of Tokyo

\section{Yanan Liu}

Department of Animal Dairy, and Veterinary Sciences, College of Agriculture and Applied Sciences, Utah State University

\section{Deanna Larson}

epartment of Animal Dairy, and Veterinary Sciences, College of Agriculture and Applied Sciences, Utah State University

\section{Jorge Osorio}

University of Wisconsin-Madison

\section{Juan Hernandez-Ortiz}

Department of Pathobiological Sciences, School of Veterinary Medicine. University of Wisconsin, Madison

\section{Karl Čiuoderis}

Universidad Nacional de Colombia

\section{Kelsey Florek}

Wisconsin State Laboratory of Hygiene

\section{Mit Patel}

Center for Childhood Infections and Vaccines of Children's Healthcare of Atlanta, Department of Pediatrics, Emory Vaccine Center, Emory University School of Medicine

\section{Allen Bateman}

Wisconsin State Laboratory of Hygiene

\section{Abby Odle}

Department of Microbiology and Immunology, University of lowa. 


\section{Lok-Yin Wong}

Department of Microbiology and Immunology, University of lowa.

\section{Zhongde Wang}

Utah State University https://orcid.org/0000-0003-2441-4729

\section{Venkata Viswanadh Edara}

Emory https://orcid.org/0000-0001-9321-7839

\section{Zhenlu Chong}

Washington University

\section{Larissa Thackray}

Washington University in St. Louis School of Medicine https://orcid.org/0000-0002-9380-6569

\section{Hiroshi Ueki}

The University of Tokyo https://orcid.org/0000-0002-6557-0771

\section{Seiya Yamayoshi}

University Of Tokyo https://orcid.org/0000-0001-7768-5157

\section{Masaki Imai}

University of Tokyo https://orcid.org/0000-0001-6988-1975

\section{Stanley Perlman}

University of lowa https://orcid.org/0000-0003-4213-2354

\section{Richard Webby}

St. Jude Children's Research Hospital https://orcid.org/0000-0002-4397-7132

\section{Robert Seder}

National Institutes of Health https://orcid.org/0000-0003-3133-0849

\section{Mehul Suthar}

Emory University School of Medicine

\section{Adolfo Garcia-Sastre}

Icahn School of Medicine at Mount Sinai https://orcid.org/0000-0002-6551-1827

\section{Michael Schotsaert}

Icahn School of Medicine at Mount Sinai https://orcid.org/0000-0003-3156-3132

\section{Tadaki Suzuki}

National Institute of Infectious Diseases https://orcid.org/0000-0002-3820-9542

\section{Adrianus Boon}

Washington University https://orcid.org/0000-0002-4700-8224

\section{Yoshihiro Kawaoka}

University of Wisconsin-Madison https://orcid.org/0000-0001-5061-8296

\section{Daniel Douek}

$\mathrm{NIH}$

\section{Juan Moliva}

Vaccine Research Center, $\mathrm{NIH}$

\section{Nancy Sullivan}


Vaccine Research Centre, NIH

\section{Matthew Gagne}

VRC/NIAID/NIH

\section{Amy Ransier}

National Institutes of Health

\section{James Case}

Washington University School of Medicine https://orcid.org/0000-0001-7331-5511

\section{Trushar Jeevan}

\section{John Franks}

St. Jude Children's Research Hospital, Memphis, TN, USA

\section{Thomas Fabrizio}

St Jude Children's Research Hospital https://orcid.org/0000-0002-8960-0728

\section{Jennifer DeBeauchamp}

Department of Infectious Diseases, St. Jude Children's Research Hospital

\section{Lisa Kercher}

Department of Infectious Diseases, St. Jude Children's Research Hospital, Memphis, TN, USA

\section{Patrick Seiler}

Department of Infectious Diseases, St. Jude Children's Research Hospital

\section{Gagandeep Singh}

https://orcid.org/0000-0003-3260-2631

\section{Prajakta Warang}

Icahn School of Medicine at Mount Sinai

\section{Ana S. Gonzalez-Reiche}

Icahn School of Medicine at Mount Sinai https://orcid.org/0000-0003-3583-4497

\section{Emilia Sordillo}

Icahn School of Medicine at Mount Sinai

\section{Harm van Bakel}

Icahn School of Medicine at Mount Sinai https://orcid.org/0000-0002-1376-6916

\section{Viviana Simon}

Icahn School of Medicine at Mount Sinai https://orcid.org/0000-0002-6416-5096

\section{Biological Sciences - Article}

\section{Keywords:}

Posted Date: December 29th, 2021

DOI: https://doi.org/10.21203/rs.3.rs-1211792/v1 
License: (c) (i) This work is licensed under a Creative Commons Attribution 4.0 International License. Read Full License

Version of Record: A version of this preprint was published at Nature on January $21 \mathrm{st}, 2022$. See the published version at https://doi.org/10.1038/s41586-022-04441-6. 


\section{Abstract}

Despite the development and deployment of antibody and vaccine countermeasures, rapidly-spreading SARS-CoV-2 variants with mutations at key antigenic sites in the spike protein jeopardize their efficacy. The recent emergence of B.1.1.529, the Omicron variant1,2, which has more than 30 mutations in the spike protein, has raised concerns for escape from protection by vaccines and therapeutic antibodies. A key test for potential countermeasures against B.1.1.529 is their activity in pre-clinical rodent models of respiratory tract disease. Here, using the collaborative network of the SARS-CoV-2 Assessment of Viral Evolution (SAVE) program of the National Institute of Allergy and Infectious Diseases (NIAID), we evaluated the ability of multiple B.1.1.529 Omicron isolates to cause infection and disease in immunocompetent and human ACE2 (hACE2) expressing mice and hamsters. Despite modeling and binding data suggesting that B.1.1.529 spike can bind more avidly to murine ACE2, we observed attenuation of infection in 129, C57BL/6, and BALB/c mice as compared with previous SARS-CoV-2 variants, with limited weight loss and lower viral burden in the upper and lower respiratory tracts. Although K18-hACE2 transgenic mice sustained infection in the lungs, these animals did not lose weight. In wild-type and hACE2 transgenic hamsters, lung infection, clinical disease, and pathology with B.1.1.529 also were milder compared to historical isolates or other SARS-CoV-2 variants of concern. Overall, experiments from multiple independent laboratories of the SAVE/NIAID network with several different B.1.1.529 isolates demonstrate attenuated lung disease in rodents, which parallels preliminary human clinical data.

\section{Introduction}

Severe acute respiratory syndrome coronavirus 2 (SARS-CoV-2) has caused the global Coronavirus Disease 2019 (COVID-19) pandemic resulting in millions of deaths worldwide. The extensive morbidity and mortality associated with the COVID-19 pandemic made the development of SARS-CoV-2 vaccines, antibody-based countermeasures, and direct acting antiviral agents a global health priority. As part of the development process, several key animal models of SARS-CoV-2 infection and lung pathogenesis were developed in mice, hamsters, nonhuman primates (NHP) and other animals for rapid testing and evaluation ${ }^{3}$. Remarkably, several highly effective vaccines and antibody therapeutics targeting SARSCoV-2 spike protein gained regulatory approval and were deployed with hundreds of millions of doses given worldwide (https://covid19.who.int). While these measures markedly reduced numbers of infections, hospitalizations, and deaths, their efficacy has been jeopardized by emergence of highly transmissible variant SARS-CoV-2 strains with mutations in the spike protein that could compromise protective immune responses and therapeutics.

Currently available vaccines and antibody countermeasures were developed using the SARS-CoV-2 spike protein from strains circulating during the early phases of the pandemic in 2020. The SARS-CoV-2 spike protein engages angiotensin-converting enzyme 2 (ACE2) on the surface of human cells to facilitate entry and infection of cells ${ }^{4}$. Upon cell attachment, SARS-CoV-2 spike proteins are cleaved by host proteases into S1 and S2 fragments. The S1 protein includes the N-terminal (NTD) and receptor binding (RBD) 
domains, whereas the S2 protein promotes membrane fusion. The RBD, in particular, is the target of many potently neutralizing monoclonal ${ }^{5-9}(\mathrm{mAb})$ and serum polyclonal antibodies ${ }^{10}$. Although SARS-CoV-2 spike proteins from strains early in the pandemic bound to ACE2 from multiple susceptible animal species (e.g., hamster, ferret, and NHP), they did not bind mouse ACE2, which explained why conventional laboratory strains of mice could not be infected efficiently by SARS-CoV-24,11; indeed, mice could become susceptible through ectopic expression of hACE2 via a transgene ${ }^{12-14}$, adenoviral vector delivery ${ }^{15,16}$, or expression of hACE2 by the mouse ACE2 promoter ${ }^{17-19}$. However, later in the pandemic, several variant strains (e.g., B.1.1.7 (Alpha), B.1.351 (Beta), B.1.1.28 (Gamma), and B.1.621 (Mu)) acquired a mouse-adapting spike mutation (N501Y), which allowed engagement of murine ACE2 and productive infection of laboratory strains of mice without ectopic hACE2 expression ${ }^{20-22}$.

In late November of 2021, the Omicron (B.1.1.529) variant emerged, which has the largest number (>30) of mutations, deletions, or insertions in the spike protein described to date. This number of changes in the spike has raised concerns for escape from protection by vaccines and therapeutic mAbs. Most B.1.1.529 isolates have a constellation of changes in the spike protein including: A67V, D69-70, T95I, G142D, D143145, D211, L212I, insertion 214EPE, G339D, S371L, S373P, S375F, K417N, N440K, G446S, S477N, T478K, E484A, Q493R, G496S, Q498R, N501Y, Y505H, T547K, D614G, H655Y, N679K, P681H, N764K, D796Y, N856K, Q954H, N969K, and L981F. The presence of the N501Y mutation along with additional mutations (K417, E484, Q493, Q498, and N501) at sites associated with mouse adaptation by serial passage 23-28 $^{2}$ suggested that B.1.1.529 should infect laboratory strains of mice ${ }^{29}$. Indeed, one recent study speculated that because mutations in the B.1.1.529 spike protein overlap with ones known to promote adaptation to mouse hosts, the progenitor of B.1.1.529 jumped from humans to mice, and then back into humans ${ }^{30}$. In support of this, B.1.1.529 but not Wuhan-1 RBD binds to murine ACE2 ${ }^{31}$. Lastly, hamsters have been a valuable pre-clinical animal model for assessing countermeasures against SARS-CoV2 and variants. Hamsters are highly susceptible to SARS-CoV-2 infection with similar pathological changes seen in lung tissues from COVID-19 patients ${ }^{3,32,33}$. Here, using experimental data from multiple laboratories of the SAVE/NIAID consortium, we report on the infectivity of multiple B.1.1.529 isolates in mice and hamsters, two key rodent models of SARS-CoV-2 infection and pathogenesis that are used to model human disease and evaluate countermeasures.

\section{Results}

B.1.1.529 infection in mice. Based on the presence of several mutations that are considered mouseadapting, we predicted that B.1.1.529 might productively infect immunocompetent mice and cause lung disease as seen with other recombinant strains (WA1/2020 N501Y/D614G) or variants (e.g., B.1.351) containing N501Y mutations. We first tested B.1.1.529 in 129 mice. Three laboratories in the SAVE/NIAID consortia (M.S.D. [St. Louis], M.S.S. [Atlanta], and A.G.S. [New York]) independently inoculated 6 to 8week-old (A.G.S.) or 10 to 20-week-old (M.S.D and M.S.S.) 129 mice with $10^{4}, 10^{5}$ or $10^{6}$ infectious units (plaque- [PFU] or focus-forming [FFU] units) of three different B.1.1.529 strains (isolated in Wisconsin, Georgia, and New York). At 3 to 4 days-post-infection (dpi) with SARS-CoV-2 strains encoding N501Y 
substitution (e.g. WA1/2020 N501Y/D614G or B.1.351), 129 mice sustain 10 to $15 \%$ loss of body weight. However, after inoculation of B.1.1.529, 129 mice failed to lose weight in all three laboratories (Fig 1a). Similarly, aged (10 to 14-month-old) C57BL/ 6 breeders also did not lose significant weight after B.1.1.529 infection, whereas those infected with B.1.351 did (Fig 1a).

We next measured the viral burden in the upper and lower respiratory tract of B.1.1.529-infected 129 mice and compared this to side-by-side infection with B.1.351 or historical data with WA1/2020 N501Y/D614G ${ }^{22,34}$. At 3 or 4 dpi, 129 mice infected with WA1/2020 N501Y/D614G or B.1.351 sustained high levels of infection in the nasal wash, nasal turbinates, and lungs (Fig 1 b-c); this level of infection previously was associated with histological evidence of mild pneumonia20,22,34. At 3 dpi, B.1.1.529 infection in the nasal turbinates and lungs was 10 to 100 -fold lower than in B.1.351-infected mice $(P<$ 0.01, Fig 1c). At 4 dpi, in contrast to WA1/2020 N501Y/D614G infection, we were unable to detect B.1.1.529 viral RNA in the nasal wash. Moreover, at this time point, 10 to 100 -fold lower levels of viral RNA were present in lung homogenates of B.1.1.529-infected mice compared to WA1/2020 N501Y/D614G-infected mice (Fig 1b). Based on weight change and viral burden, B.1.1.529 appeared attenuated in 129 mice.

Members of the SAVE/NIAID group (Y.K.) also tested B.1.1.529 in BALB/c mice and compared this to infection with B.1.351 variant. At $2 \mathrm{dpi}$, infectious virus levels in the nasal turbinates and lungs were significantly lower $(\sim 1,000$-fold, $P<0.001)$ in BALB/C mice infected with B.1.1.529 compared to B.1.351 virus (Fig 1d). We next used a whole-body plethysmography system ${ }^{35}$ to measure pulmonary function in infected BALB/c mice. At $2 \mathrm{dpi}$, whereas B.1.351 caused an increase $(P<0.001)$ in the lung enhanced pause (Penh), a surrogate marker for bronchoconstriction or airway obstruction, B.1.1.529 did not (Fig 1e). Consistent with this result, the ratio of peak expiratory flow (Rpef) was decreased at $2 \mathrm{dpi}$ in BALB/C mice infected with B.1.351 but not B.1.1.529 $(P<0.001$, Fig 1e). Thus, based on multiple parameters, lung infection and disease after B.1.1.529 was attenuated compared to other strains.

Given the attenuation in several strains of conventional laboratory mice, two groups tested B.1.1.529 infection in 5 to 6-month-old (M.S.D. and A.G.S.) K18-hACE2 transgenic mice, which express hACE2 under an epithelial cytokeratin promoter ${ }^{12}$, and are more susceptible to SARS-CoV-2 infection and disease ${ }^{14}$. At intranasal inoculating doses ranging from $10^{3}$ to $10^{5}$ infectious units of B.1.1.529, weight loss was not observed over the first 5 to 6 days of infection in younger or older K18-hACE2 mice (Fig 1f). These data contrasts with historical results with WA1/2020 D614G or variant (e.g., B.1.351) SARS-CoV-2 strains $^{14,22,34,36}$, which uniformly induce weight loss starting at $4 \mathrm{dpi}$. Although we observed clinical attenuation of B.1.1.529 in K18-hACE2 mice, the virus nonetheless accumulated in the upper and lower respiratory tract (Fig $\mathbf{1 g})$.

B.1.1.529 infection in hamsters. Because Syrian hamsters are a pivotal model for studying SARS-CoV-2 pathogenesis and evaluating countermeasures ${ }^{3}$, four members of our SAVE/NIAID team (R.A.S., A.C.M.B, R.J.W., and Y.K.) tested three different B.1.1.529 strains (from Wisconsin, Georgia, and Japan) for their ability to infect and cause disease. Whereas intranasal infection with historical or other variant SARS- 
CoV-2 strains generally resulted in $\sim 10$ to $15 \%$ reduction in body weight over the first week, we observed no weight loss in any of the hamsters inoculated with B.1.1.529, regardless of inoculating dose (Fig 2a-e). However, animals infected with B.1.1.529 did not gain body weight as rapidly as uninfected hamsters. Viral RNA analysis at 4 dpi showed lower levels of B.1.1.529 infection in the nasal wash (2-fold, $P<0.05$ ) and lungs (12-fold, $P<0.001$ ) compared to WA1/2020 D614G ([A.C.M.B.]; Fig 2f). A comparison of infectious viral burden in tissues at 3 dpi between B.1.617.2 (Delta) and B.1.1.529 (Omicron) viruses [Y.K.] showed virtually no difference in nasal turbinates but substantially less infection of B.1.1.529 in the lungs of most animals (Fig 2g). A comparison of viral RNA levels between WA1/2020 and B.1.1.529 viruses (R.J.W) in nasal washes at 4 dpi did not show substantial differences in nasal wash titers (Fig 2h). Thus, in hamsters, upper, but not lower, respiratory tract infection by B.1.1.529 appears relatively intact.

We also used whole-body plethysmography to measure pulmonary function in infected Syrian hamsters (Y.K.). Starting at $3 \mathrm{dpi}$ and continuing until $7 \mathrm{dpi}$, infection with B.1.617.2 caused an increase $(P<0.05)$ in the lung enhanced pause (Penh), whereas B.1.1.529 infection did not (Fig 2i). Consistent with this result, the ratio of peak expiratory flow (Rpef) was decreased at 5 and $7 \mathrm{dpi}$ in animals infected with B.1.617.2 but not B.1.1.529 ( $P<0.001$, Fig 2i). Finally, hamsters infected with B.1.617.2, but not B.1.1.529, demonstrated a decrease in respiratory rate compared to uninfected control animals. Thus, based on multiple functional parameters, lung infection and disease after B.1.1.529 infection was attenuated compared to other variant strains.

We next performed microcomputed tomography (micro-CT) to assess for lung abnormalities in hamsters at $7 \mathrm{dpi}$. We used a previously defined CT severity score (see Legend and Methods) to evaluate animals for nodules, ground glass opacities, and regions of lung consolidation ${ }^{35}$. Micro-CT analysis revealed lung abnormalities in all B.1.617.2- infected hamsters on $7 \mathrm{dpi}$ that were consistent with commonly reported imaging features of COVID-19 pneumonia ${ }^{37}$. In comparison, analysis of B.1.1.529-infected hamsters on 7 dpi revealed patchy, ill-defined ground glass opacity consistent with minimal to mild pneumonia. Accordingly, Syrian hamsters infected with B.1.617.2 had a high CT disease score ( 12), whereas those infected with B.1.1.529 had a substantially lower disease score $(\sim 2)$ that was only slightly greater than mock-infected hamsters (Fig 2j-k).

We also compared lung pathology in Syrian hamsters after infection with B.1.617.2 or B.1.1.529 viruses. Macroscopically, the lungs obtained from the B.1.617.2-infected hamsters showed congestion and/or hemorrhage, but this was absent in B.1.1.529-infected animals (Fig. 3a). At the microscopic level, immune cell infiltration and inflammation were present in the peribronchial regions of the lungs at 3 dpi with B.1.617.2. Moreover, at 6 days post B.1.617.2 infection, extensive infiltration of neutrophils and lymphocytes in the alveolar space and walls was accompanied by focal pulmonary edema and alveolar hemorrhage (Fig $\mathbf{3 b}$, inset), and regenerative changes in the bronchial epithelia became prominent (Fig 3b). In contrast, in B.1.1.529-infected hamsters, small foci of inflammation in the alveoli and peribronchial regions were observed only at $6 \mathrm{dpi}$ (Fig 3b). A semi-quantitative histopathology severity score of viral pneumonia at $6 \mathrm{dpi}$ showed a worse score after B.1.617.2 than B.1.1.529 infection (Fig 3c). 
Consistent with these findings, after B.1.617.2 infection, viral RNA was detected readily in the alveoli and bronchial epithelia in lung tissue sections at 3 and 6 dpi (Fig 3d). In comparison, after B.1.1.529 infection, fewer bronchial epithelial cells and alveoli were positive for viral RNA at either time point (Fig 3d). Collectively, these findings suggest that the B.1.1.529 Omicron variant replicates less efficiently in the lungs of Syrian hamsters, which results in less severe pneumonia compared to the B.1.617.2 Delta variant.

Although hamsters are susceptible to SARS-CoV-2 infection without a requirement for host adaptation and show some similarities to that observed in COVID-19 patients, they develop self-limiting clinical and respiratory disease. Even though hamster ACE2 can serve as a receptor for SARS-CoV-2 spike protein, some of the contact residues in human ACE2 are not conserved ${ }^{38}$, which could diminish infectivity. Moreover, ACE2 expression levels on particular cells in the respiratory tract may differ slightly between hamsters and humans, which could impact infectivity and clinical outcome. To develop a more susceptible hamster model, members of the consortium (Y.K.) used transgenic hamsters (generated by Z.W.) expressing human ACE2 under the epithelial cytokeratin-18 promoter ${ }^{39}$. Whereas intranasal inoculation of $10^{3}$ PFU of HP-095 D614G virus resulted in marked weight loss within the first week (Fig 2I) and uniform mortality by $10 \mathrm{dpi}$ (Fig 2m), less weight loss and death $(P<0.05)$ were observed after infection with $10^{3} \mathrm{PFU}$ of B.1.1.529. Consistent with these clinical data, 1,000 to 10,000-fold lower levels of infectious virus were measured in the lungs of hACE2 transgenic hamsters challenged with B.1.1.529 compared to the HP-095 D614G virus at 3 and 5 dpi (Fig 2n). Notably, and as seen in wild-type Syrian hamsters, smaller differences in infection were observed in the nasal turbinates. Thus, B.1.1.529 infection in the lung is attenuated in both wild-type and hACE2 transgenic hamsters.

\section{Discussion}

Our experiments suggest that compared to other SARS-CoV-2 isolates (e.g., B.1.351 or B.1.617.2), the B.1.1.529 Omicron variant infection is attenuated in laboratory mice and hamsters for causing infection and/or disease. While these results are consistent with the very preliminary clinical data in humans suggesting that B.1.1.529 causes a more transmissible yet possibly milder respiratory infection ${ }^{40,41}$, the basis for the attenuation in rodents remains unknown. One pre-print study suggests that B.1.1.529 replicates faster in the human bronchus and less in lung cells, which may explain its greater transmissibility and putative lower disease severity ${ }^{42}$; although it remains unclear if these observations extend to rodents, we observed less infection of hamster bronchial cells in vivo with B.1.1.529 Omicron than B.1.617.2 Delta virus. We also measured lower viral burden in nasal washes and turbinates in 129 mice compared to other SARS-CoV-2 strains. The attenuation in mice was unexpected given that B.1.1.529 has multiple mutations in the RBD that are sites (K417, E484, Q493, Q498, and N501) associated with adaptation for mice $23-25$. Moreover, the attenuation in hamsters also was surprising, given that all prior SARS-CoV-2 variants have replicated relatively equivalently and to high levels in this animal $^{35,43,44}$. However, our results showing attenuation of B.1.1.529 in hamsters are consistent with another preliminary report ${ }^{45}$. Whereas the more than 30 substitutions (mutations, deletions, and 
insertions) in the B.1.1.529 could impact receptor engagement and cell entry, sequence changes in other structural, non-structural, and immune evasion proteins could affects replication, temperature sensitivity, cell-to-cell spread, cell and tissue tropism, dissemination, and induction of pro-inflammatory immune responses in a species-specific manner. Thus, detailed genetic and functional studies are required to define the basis of virological and clinical attenuation of B.1.1.529 in mice and hamsters.

Although we observed clinical attenuation of B.1.1.529 in mice and hamsters, these animals likely will still have utility in evaluating vaccine, antibody, or small molecule inhibitors. All of the mice and hamsters tested, to varying degrees, showed evidence of viral replication and dissemination to the lower respiratory tract, which could be prevented or mitigated by prophylactic or therapeutic countermeasures. The most severe B.1.1.529 infection and disease was observed in hACE2 expressing mice and hamsters, which is consistent with results with other SARS-CoV-2 strains and variants ${ }^{14,22,39,46}$, and possibly related to the enhanced interactions between hACE2 and the B.1.1.529 spike protein ${ }^{47}$.

These in vivo studies were performed as part of the SAVE/NIAID consortium and reflect a collaborative network of expert investigators that communicates multiple times per week to expedite progress on emerging SARS-CoV-2 variants that threaten vaccine efficacy. Several key advantages with relevance to small animal models arise from this interactive format: (a) Animal experiments were reproduced across laboratories providing immediate confidence in results and interpretation; (b) Multiple independent B.1.1.529 isolates were used limiting the possibility of skewing of particular sequence adaptations in a particular strain from one laboratory that could impact results; (c) Multiple strains and vendors of mice (129, BALB/c, C57BL/6, and K18-hACE2) and hamsters (Syrian Golden and hACE2 transgenic) at different ages were tested in a short time span allowing for the accumulation of a robust and larger data set and an appreciation of the generality of the results; and (d) The groups used an overlapping set of metrics (weight measurements, viral load, plethysmography, histopathology, and micro-CT imaging) to evaluate and compare infection and disease in the different animal models.

Nonetheless, we note several limitations to the conclusions of our study: (1) While our experiments, showing attenuation of B.1.1.529 in mice and hamsters, correlate with preliminary clinical data in humans, further experiments in NHPs and evaluation of human data are needed to corroborate these findings. Moreover, all of our studies ended at relatively early time points to allow for acquisition of respiratory tract tissues and titration of virus. It is possible that B.1.1.529 has altered tropism for other organs in rodents or requires additional time for replication and dissemination; (2) In all studies, we used the prevailing B.1.1.529 Omicron isolate that lacks an R346K mutation. While $~ 8 \%$ of B.1.1.529 sequences in GISAID currently have an R346K mutation, this substitution or others in genes apart from spike might further affect virulence in rodents. Although one of the B.1.1.529 isolates we tested (A.G.S., hCoV-19/USA/NY-MSHSPSP-PV44476/2021) contains an additional A701V mutation in spike near the furin cleavage site, it was still attenuated in mice compared to the B.1.351 virus; (3) Our data are focused on studying infection in a given animal and do not address other key questions in the field including vaccine protection, transmission, or antibody or small molecule drug protection; and (4) Due to time constraints, detailed pathological analyses were not performed for all of the animal species studied. 
In summary, our studies by a network of investigators at multiple sites with several independently isolated B.1.1.529 strains rapidly and reproducibly demonstrated attenuated infection in multiple strains of laboratory mice and hamsters. Despite the attenuation of B.1.1.529 Omicron variants in causing infection in rodents, some of these pre-clinical models likely will retain utility for testing existing and novel vaccines and therapeutics given that viral replication occurs and some inflammatory and clinical disease is observed. Studies are ongoing to determine the basis for attenuation in mice and hamsters and to determine how this relates to the patterns of B.1.1.529 Omicron infection seen in humans.

\section{Declarations}

\section{ACKNOWLEDGEMENTS}

This study was supported by grants and contracts from NIH (R01 Al157155 [M.S.D], U01 Al151810 [M.S.D.], 75N93021C00014 (Center for Research on Influenza Pathogenesis and Transmission (CRIPT)) [Y.K. and A.G.S.], HHSN272201400008C [Y.K.], HHSN272201700041I [Z.W.], 75N93020F00001/A38 [Z.W.], P51OD011132 [M.S.S.], R56Al147623 [M.S.S.], HHSN272201400004C [M.S.S.], 75N93021C00017 [M.S.S.], P01 Al060699 [S.P.], R01 Al129269 [S.P.], and 75N93019C00051 [M.S.D.], 75N93021C00016 (St. Jude Center of Excellence on Influenza Research and Response (CEIRR)) [R.J.W. and A.C.M.B.]), and grants from the Research Program on Emerging and Re-emerging Infectious Diseases (JP20fk0108412, JP21fk0108615, JP20fk0108472, and JP21fk0108104), a Project Promoting Support for Drug Discovery (JP20nk0101632), the Japan Program for Infectious Diseases Research and Infrastructure (JP21wm0125002) from the Japan Agency for Medical Research and Development (AMED). The Woodruff Health Sciences Center and Emory School of Medicine, Woodruff Health Sciences Center 2020 COVID-19 CURE Award also supported this study. The piggyBac vector, pmhyGEMIE-3, was a gift from Dr. Stefan Moisyadi at the University of Hawaii. The Mount Sinai Pathogen Surveillance (E.M.S., H.v.B., V.S.) is supported by institutional school and hospital funds as well as by an option to 75N93021C00014 (A.G.S.). We thank Dr. Randy Albrecht for support with the BSL3 facility and procedures at the Icahn School of Medicine at Mount Sinai, New York.

\section{AUTHOR CONTRIBUTIONS}

P.J.H., S.I., K.I-H., T.M., M.K., S.M.S., T.D., A.J., S.L.F., B.Y., B.W., K.F., M.U., N.N., M.Ito, R.W., R.U., R.L., Y.L., D.L., J.P.H-O., K.C., K.F., M.P., A.O., L-Y. W., A.B., V.V.E., Z.C., H.U., M.S., T.S., K.C., J.B.C., J.F., T.F., T.J., P.S., J.D., G.S., P.W. and L.K. performed the infection experiments in mice and hamsters, titrated virus in tissues, and analyzed pathology. R.L., Y.L., D.L. and Z.W. generated human ACE2 hamsters. E.O., J.P.H-O., K.C., K.F., A.C.B., D.C.D., P.J.H., G.S., M.G., Y.S-T., and A.R.H. generated, propagated, and/or sequenced viruses. S.L. analyzed the micro-CT images. A.S.G.R. and H.v.B. performed screening and whole virus genome analysis. E.M.S., H.v.B., and V.S. planned the viral surveillance and analyzed data. The PSP study group contributed to the rapid accessioning and transfer of nasopharyngeal swabs, the extraction of RNA and sequencing allowing for the quick identification of B.1.1.529 in New York City. Y.K., M.S.S., A.G.S., R.A.S., R.J.W., J.E.O., Z.W., H.U., S.Y., M.I., N.J.S., L.B.T., T.Z., A.C.M.B., and M.S.D. obtained funding, 
conceived of the study, and supervised research. A.C.M.B., Y.K., and M.S.D. wrote the initial draft, with all other authors providing editorial comments.

\section{COMPETING INTERESTS}

M.S.D. is a consultant for Inbios, Vir Biotechnology, Senda Biosciences, and Carnival Corporation, and on the Scientific Advisory Boards of Moderna and Immunome. The Diamond laboratory has received funding support in sponsored research agreements from Moderna, Vir Biotechnology, and Emergent BioSolutions. The Boon laboratory has received unrelated funding support in sponsored research agreements from Al Therapeutics, GreenLight Biosciences Inc., and Nano targeting \& Therapy Biopharma Inc. M.S.S serves on Advisory boards for Moderna and Ocugen. Y.K. has received unrelated funding support from Daiichi Sankyo Pharmaceutical, Toyama Chemical, Tauns Laboratories, Inc., Shionogi \& Co. LTD, Otsuka Pharmaceutical, KM Biologics, Kyoritsu Seiyaku, Shinya Corporatoin, and Fuji Rebio. The A.G.S. laboratory has unrelated received research support from Pfizer, Senhwa Biosciences, Kenall Manufacturing, Avimex, Johnson \& Johnson, Dynavax, 7Hills Pharma, Pharmamar, ImmunityBio, Accurius, Nanocomposix, Hexamer, N-fold LLC, Model Medicines, Atea Pharma and Merck. A.G.-S. has paid or equity-based consulting agreements for the following companies: Vivaldi Biosciences, Contrafect, 7Hills Pharma, Avimex, Vaxalto, Pagoda, Accurius, Esperovax, Farmak, Applied Biological Laboratories, Pharmamar, Paratus, CureLab Oncology, CureLab Veterinary and Pfizer, outside of the reported work. A.G.$S$. is inventor on patents and patent applications on the use of antivirals and vaccines for the treatment and prevention of virus infections and cancer, owned by the Icahn School of Medicine at Mount Sinai, New York, outside of the reported work. Icahn School of Medicine at Mount Sinai has filed patent applications relating to SARS-CoV-2 serological assays that list Viviana Simon as co-inventor. The remaining authors declare no competing interests.

\section{Methods}

Cells. Vero-TMPRSS $2^{35,48,49}$ and Vero-hACE2-TMPRSS $2^{50}$ cells were cultured at $37^{\circ} \mathrm{C}$ in Dulbecco's Modified Eagle medium (DMEM) supplemented with 10\% fetal bovine serum (FBS), $10 \mathrm{mM} \mathrm{HEPES} \mathrm{pH}$ 7.3 , and $100 \mathrm{U} / \mathrm{ml}$ of penicillin-streptomycin. Vero-TMPRSS2 cells were supplemented with $5 \mathrm{mg} / \mathrm{mL}$ of blasticidin or $1 \mathrm{mg} / \mathrm{mL}$ of geneticin (depending on the cell line) and in some cultures with plasmocin. Vero-hACE2-TMPRSS2 cells were supplemented with $10 \mu \mathrm{g} / \mathrm{mL}$ of puromycin. All cells routinely tested negative for mycoplasma using a PCR-based assay.

Viruses. The WA1/2020 recombinant strains with substitutions (D614G and/or N501Y/D614G) were described previously ${ }^{51}$. The B.1.1.529 isolates (hCoV-19/USA/WI-WSLH-221686/2021 [Y.K., M.S.D., R.A.S., A.C.M.B., GISAID: EPI_ISL_7263803], hCoV-19/Japan/NC928-2N/2021 (NC928) [Y.K., GISAID: EPI_ISL_7507055], hCoV-19/USA/NY-MSHSPSP-PV44476/2021 [A.G.S., GISAID:

EPI_ISL_7908052], and hCoV19/EHC_C19_2811C [M.S.S., S.P., and R.J.W. GISAID: EPI_ISL_7171744]) were obtained from nasal swabs and passaged on Vero-TMPRSS2 cells as described $33,35,49$. Other viruses used included: SARS-CoV-2/UT-NC002-1T/Human/2020/Tokyo 
(NC002 ${ }^{33}$ ), SARS-CoV-2/UT-HP095-1N/Human/2020/Tokyo (HP-095; D614G), hCoV-

19/USA/CA_CDC_5574/2020 (Alpha, B.1.1.7; BEI NR54011), hCoV-19/USA/MD-HP01542/2021 (Beta, B.1.351; HP01542), 20H/501Y.V2 (Beta, B.1.351), hCoV-19/USA/WI-UW-5250/2021 (Delta, B.1.617.2; UW5250) ${ }^{52}$, hCoV-19/USA/CA-VRLC009/2021 (Epsilon, B.1.427; VRLC009), hCoV-19/USA/NYCPV26425/2021 (B.1.526, lota; PV26425), hCoV-19/USA/217-80384/2021 (B.1.621, Mu; 80384), and hCoV-19/Colombia/SEC0506/2021 (C.37, Lambda; SEC0506). All viruses were subjected to nextgeneration sequencing as described ${ }^{53}$ to confirm the stability of substitutions and avoid introduction of adventitious mutations. All virus experiments were performed in an approved biosafety level 3 (BSL-3) facility.

Animal experiments and approvals. Animal studies were carried out in accordance with the recommendations in the Guide for the Care and Use of Laboratory Animals of the National Institutes of Health. The protocols were approved by the Institutional Animal Care and Use Committee at the Washington University School of Medicine (assurance number A3381-01), University of Wisconsin, Madison (V006426), St. Jude Children's Research Hospital (Assurance number D16-00043), Emory University, University of lowa (assurance number A3021-01), Icahn School of Medicine at Mount Sinai (PROT0202100007), BIOQUAL, Inc., and the Animal Experiment Committee of the Institute of Medical Science, the University of Tokyo (approval numbers PA19-72 and PA19-75). Virus inoculations were performed under anesthesia that was induced and maintained with ketamine hydrochloride and xylazine, and all efforts were made to minimize animal suffering.

Mouse infection experiments. Heterozygous K18-hACE2 C57BL/6J mice (strain 2B6.Cg-Tg(K18ACE2)2Prlmn/J), 129 mice (strain: 129S2/SvPasCrl or 129S1/SvImJ), and C57BL/6 (strain 000664) mice were obtained from The Jackson Laboratory and Charles River Laboratories. BALB/c mice were purchased from Japan SLC Inc. Animals were housed in groups and fed standard chow diets. Infection experiments were performed as follows: (a) [M.S.D] 16- to 17-week-old female $129 \mathrm{~S} 2$ mice were administered $10^{4}$ or $10^{5} \mathrm{FFU}$ of B.1.1.529 [hCoV-19/USA/WI-WSLH-221686/2021] or other SARS-CoV-2 strains by intranasal administration; 5-month-old female K18-hACE2 mice were inoculated by intranasal route with $10^{3}, 10^{4}$ or $10^{5} \mathrm{FFU}$ of SARS-CoV-2. (b) [M.S.S.] 129S1 male and female mice were used were between 10-20 weeks of age. Mice were anesthetized with isoflurane and inoculated intranasally with virus (50 mL, $10^{6} \mathrm{PFU} /$ mouse); (c) [Y.K.] Six-week-old female BALB/c mice were inoculated intranasally with $10^{5} \mathrm{PFU}$ of hCoV-19/Japan/NC928-2N/2021 or hCoV-19/USA/MD-HP01542/2021; (d) [S.P.] Retired breeder female C57BL/ 6 mice (10 to 14-month-old) were anesthetized with ketamine-xylazine and inoculated intranasally with SARS-CoV-2 in a total volume of $50 \mu \mathrm{L}$ of DMEM. Animal weight and health were monitored daily; and (e) [A.G.S.] 10- or 31-week-old female $129 \mathrm{~S} 1$ mice and 6-month-old female K18hACE2 mice were inoculated by intranasal route under light ketamine/xylazine sedation with $10^{4} \mathrm{PFU}$ of hCoV-19/USA/NY-MSHSPSP-PV44476/2021 in a total volume of $50 \mu \mathrm{L}$.

Hamster infection experiments. Five-to-six-week-old male hamsters were obtained from Charles River Laboratories, Envigo, or Japan SLC Inc. The K18-hACE2 transgenic hamster line was developed with a 
piggyBac-mediated transgenic approach, in which the K18-hACE2 cassette from the pK18-hACE2 plasmid ${ }^{12}$ was transferred into a piggyBac vector, pmhyGENIE- ${ }^{54}$, for pronuclear injection. hACE2 transgenic hamsters will be described in detail elsewhere ${ }^{39}$. Twelve-month-old transgenic female animals were used. Infection experiments were performed as follows: (a) [A.C.M.B] animals were challenged via intranasal route with $10^{3}$ PFU of WA1/2020 D614G or B.1.1.529 variant in $100 \mu \mathrm{L}$; (b) [Y.K.] Under isoflurane anesthesia, wild-type Syrian hamsters were intranasally inoculated with $10^{3} \mathrm{PFU}$ or with $10^{5}$ PFU of SARS-CoV-2 strains in $30 \mu \mathrm{L}$. Body weight was monitored daily. For virological and pathological examinations, four hamsters per group were euthanized 3 and $6 \mathrm{dpi}$, and nasal turbinates and lungs were collected. The virus titers in the nasal turbinates and lungs were determined by plaque assays on Vero-TMPRSS2 cells. Human ACE2 transgenic hamsters were intranasally inoculated with $10^{3}$ PFU of HP-095 D614G or B.1.1.529 [hCoV-19/USA/WI-WSLH-221686/2021] in $50 \mu \mathrm{L}$. Body weight and survival were monitored daily, and nasal turbinates and lungs were collected at 3 and $5 \mathrm{dpi}$ for virological analysis; (c) [R.A.S.] Six-week-old male Syrian golden hamsters were randomized into groups of 4 to 6 and inoculated with SARS-CoV-2 via delivery of $100 \mu \mathrm{L}$ of appropriately diluted virus in PBS equally split between both nostrils. Weight change and clinical observations were collected daily; and (d) [R.J.W] while under isoflurane anesthesia, male 8-10 week old hamsters were inoculated intranasally with $10^{4}$ PFU of WA1/2020 or B.1.1.529 in $100 \mu \mathrm{L}$ volume. Body weight and survival were monitored daily. Nasal washes were taken at $4 \mathrm{dpi}$ for virological analysis.

Measurement of viral burden. (a) Mouse studies. [M.S.D.] Tissues were weighed and homogenized with zirconia beads in a MagNA Lyser instrument (Roche Life Science) in $1000 \mu \mathrm{L}$ of DMEM medium supplemented with $2 \%$ heat-inactivated FBS. Tissue homogenates were clarified by centrifugation at $10,000 \mathrm{rpm}$ for $5 \mathrm{~min}$ and stored at $-80^{\circ} \mathrm{C}$. RNA was extracted using the MagMax mirVana Total RNA isolation kit (Thermo Fisher Scientific) on the Kingfisher Flex extraction robot (Thermo Fisher Scientific). Viral RNA (Ngene) was reverse transcribed and amplified using the TaqMan RNA-to-CT 1-Step Kit (Thermo Fisher Scientific), and data were analyzed and normalized as described previously ${ }^{55}$. [Y.K.] The viral titers in the nasal turbinates and lungs were determined by plaque assay on Vero-TMPRSS 2 cells as previously published ${ }^{49}$. [M.S.S.] At the indicated day post-infection, mice were euthanized with isoflurane overdose and one lobe of lung tissue was collected in an Omni Bead ruptor tube filled with Tri Reagent (Zymo, \#R2050-1-200). Tissue was homogenized using an Omni Bead Ruptor 24 (5.15 ms, 15 seconds), then centrifuged to remove debris. RNA was extracted using a Direct-zol RNA MiniPrep Kit (Zymo, \# R2051), then converted to cDNA using a High-capacity Reverse Transcriptase cDNA Kit (Thermo, \#4368813). SARS-CoV-2 RNA-dependent RNA polymerase and subgenomic RNA were measured as described ${ }^{27,56}$. [M.S.S.] The subgenomic SARS-CoV-2 RNA levels were quantified in nasal turbinates and lungs by qRT-PCR as previously published 27,53 .

(b) Hamster studies. [A.C.M.B.] Lungs were collected 4 dpi and homogenized in $1.0 \mathrm{~mL}$ DMEM, clarified by centrifugation $(1,000 \times \mathrm{g}$ for $5 \mathrm{~min})$ and stored at $-80^{\circ} \mathrm{C}$. Nasal washes were clarified by centrifugation $(2,000 \times \mathrm{g}$ for $10 \mathrm{~min})$ and the supernatant was stored at $-80^{\circ} \mathrm{C}$. To quantify viral load in lung tissue homogenates and nasal washes, RNA was extracted from $100 \mu \mathrm{L}$ samples using E.Z.N.A. ${ }^{\circledR}$ Total RNA Kit I 
(Omega) and eluted with $50 \mu \mathrm{L}$ of water. Four microliters RNA was used for real-time RT-qPCR to detect and quantify $\mathrm{N}$ gene of SARS-CoV-2 using TaqMan ${ }^{\text {TM }}$ RNA-to-CT 1-Step Kit (Thermo Fisher Scientific) as described ${ }^{57}$. [Y.K.] The virus titers in the nasal turbinates and lungs were determined by plaque assay on Vero E6 cells expressing human TMRPSS2 as previously published ${ }^{58}$. [R.J.W.] RNA was extracted from clarified nasal washes using the Qiagen RNeasy extraction kit (Qiagen, Hilden Germany) following the manufacturer's instructions. Samples were purified on the included columns and eluted in $50 \mu \mathrm{L}$ of nuclease free water. PCR was conducted using 4X TaqMan Fast Virus Master Mix (Thermo Fisher) and the HKU N-gene primer/probe set.

Plaque assay. Vero-TMPRSS2 or Vero-TMPRSS2-hACE2 cells were seeded at a density of $1 \times 10^{5}$ cells per well in 24-well tissue culture plates. The following day, medium was removed and replaced with $200 \mu \mathrm{L}$ of material to be titrated diluted serially in DMEM supplemented with $2 \%$ FBS. One hour later, $1 \mathrm{~mL}$ of methylcellulose overlay was added. Plates were incubated for $72 \mathrm{~h}$, then fixed with $4 \%$ paraformaldehyde (final concentration) in PBS for 20 min. Plates were stained with $0.05 \%$ (w/v) crystal violet in $20 \%$ methanol and washed twice with distilled, deionized water.

Micro-CT imaging. Hamsters were inoculated intranasally with $10^{3}$ PFU (in $30 \mu \mathrm{L}$ ) of B.1.1.529 (strain NC928), B.1.617.2 (UW-5250) or PBS. Lungs of the infected animals were imaged by using an in vivo micro-CT scanner (CosmoScan FX; Rigaku Corporation, Japan). Under ketamine-xylazine anesthesia, the animals were placed in the image chamber and scanned for $2 \mathrm{~min}$ at $90 \mathrm{kV}, 88 \mu \mathrm{A}, \mathrm{FOV} 45 \mathrm{~mm}$, and pixel size $90.0 \mu \mathrm{m}$. After scanning, the lung images were reconstructed by using the CosmoScan Database software of the micro-CT (Rigaku Corporation, Japan) and analyzed by using the manufacturer-supplied software. A CT severity score, adapted from a human scoring system, was used to grade the severity of the lung abnormalities ${ }^{59}$. Each lung lobe was analyzed for degree of involvement and scored from 0-4 depending on the severity: 0 (none, $0 \%$ ), 1 (minimal, $1 \%-25 \%$ ), 2 (mild, 26\%-50\%), 3 (moderate, $51 \%-75 \%$ ), or 4 (severe, $76 \%-100 \%$ ). Scores for the five lung lobes were summed to obtain a total severity score of $0-20$, reflecting the severity of abnormalities across the three infected groups. Images were anonymized and randomized; the scorer was blinded to the group allocation.

Pathology. Excised animal tissues were fixed in 4\% paraformaldehyde in PBS, and processed for paraffin embedding. The paraffin blocks were cut into 3- $\mu$ m-thick sections and mounted on silane-coated glass slides. Sections were processed for in situ hybridization using an RNA scope 2.5 HD Red Detection kit (Advanced Cell Diagnostics, Newark, California) with antisense probe targeting the nucleocapsid gene of SARS-CoV-2 (Advanced Cell Diagnostics). Specific antigen-antibody reactions were visualized by means of 3,3'-diaminobenzidine tetrahydrochloride staining using the Dako Envision system (Dako Cytomation). Lung tissue sections also were scored based on pathological changes. Scores were determined based on the percentage of alveolar inflammation in a given area of a pulmonary section collected from each animal in each group by using the following scoring system: 0 , no pathological change; 1 , affected area 
( $\leq 10 \%) ; 2$, affected area ( $<50 \%,>10 \%) ; 3$, affected area ( $\geq 50 \%)$; an additional point was added when pulmonary edema and/or alveolar hemorrhage was observed.

Data availability. All data supporting the findings of this study are available within the paper, in the Source Data, and from the corresponding author upon request. There are no restrictions in obtaining access to primary data.

Code availability. No code was used in the course of the data acquisition or analysis.

Reagent availability. All reagents described in this paper are available through Material Transfer Agreements.

Statistical analysis. The number of independent experiments and technical replicates used are indicated in the relevant Figure legends. Statistical analysis included unpaired $t$ tests, Mann-Whitney $U$ tests, and ANOVA with multiple corrections post-tests.

\section{References}

1 Callaway, E. \& Ledford, H. How bad is Omicron? What scientists know so far. Nature, doi:10.1038/d41586-021-03614-z (2021).

2 Torjesen, I. Covid-19: Omicron may be more transmissible than other variants and partly resistant to existing vaccines, scientists fear. BMJ (Clinical research ed 375, n2943, doi:10.1136/bmj.n2943 (2021).

3 Muñoz-Fontela, C. et al. Animal models for COVID-19. Nature 586, 509-515, doi:10.1038/s41586020-2787-6 (2020).

4 Letko, M., Marzi, A. \& Munster, V. Functional assessment of cell entry and receptor usage for SARSCoV-2 and other lineage B betacoronaviruses. Nature microbiology 5, 562-569, doi:10.1038/s41564-0200688-y (2020).

5 Pinto, D. et al. Cross-neutralization of SARS-CoV-2 by a human monoclonal SARS-CoV antibody. Nature 583, 290-295, doi:10.1038/s41586-020-2349-y (2020).

6 Cao, Y. et al. Potent neutralizing antibodies against SARS-CoV-2 identified by high-throughput single-cell sequencing of convalescent patients' B cells. Cell 182, 73-84, doi:10.1016/j.cell.2020.05.025 (2020).

7 Zost, S. J. et al. Rapid isolation and profiling of a diverse panel of human monoclonal antibodies targeting the SARS-CoV-2 spike protein. Nat Med 26, 1422-1427, doi:10.1038/s41591-020-0998-x (2020).

8 Barnes, C. O. et al. SARS-CoV-2 neutralizing antibody structures inform therapeutic strategies. Nature 588, 682-687, doi:10.1038/s41586-020-2852-1 (2020). 
9 Tortorici, M. A. et al. Ultrapotent human antibodies protect against SARS-CoV-2 challenge via multiple mechanisms. Science 370, 950-957, doi:10.1126/science.abe3354 (2020).

10 Rathe, J. A. et al. SARS-CoV-2 Serologic Assays in Control and Unknown Populations Demonstrate the Necessity of Virus Neutralization Testing. J Infect Dis, doi:10.1093/infdis/jiaa797 (2020).

11 Wan, Y., Shang, J., Graham, R., Baric, R. S. \& Li, F. Receptor recognition by novel coronavirus from Wuhan: An analysis based on decade-long structural studies of SARS. J Virol, doi:10.1128/jvi.00127-20 (2020).

12 McCray, P. B., Jr. et al. Lethal infection of K18-hACE2 mice infected with severe acute respiratory syndrome coronavirus. J Viro/ 81, 813-821, doi:10.1128/jvi.02012-06 (2007).

13 Jiang, R. D. et al. Pathogenesis of SARS-CoV-2 in Transgenic Mice Expressing Human AngiotensinConverting Enzyme 2. Cell, doi:10.1016/j.cell.2020.05.027 (2020).

14 Winkler, E. S. et al. SARS-CoV-2 infection of human ACE2-transgenic mice causes severe lung inflammation and impaired function. Nat Immuno/ 21, 1327-1335, doi:10.1038/s41590-020-0778-2 (2020).

15 Hassan, A. O. et al. A SARS-CoV-2 Infection Model in Mice Demonstrates Protection by Neutralizing Antibodies. Cell, doi:10.1016/j.cell.2020.06.011 (2020).

16 Sun, J. et al. Generation of a Broadly Useful Model for COVID-19 Pathogenesis, Vaccination, and Treatment. Cell 182, 734-743.e735, doi:10.1016/j.cell.2020.06.010 (2020).

17 Bao, L. et al. The pathogenicity of SARS-CoV-2 in hACE2 transgenic mice. Nature, doi:10.1038/s41586-020-2312-y (2020).

18 Sun, S. H. et al. A Mouse Model of SARS-CoV-2 Infection and Pathogenesis. Cell Host Microbe, doi:10.1016/j.chom.2020.05.020 (2020).

19 Winkler, E. S. et al. SARS-CoV-2 causes lung infection without severe disease in human ACE2 knock-in mice. J Virol, Jvi0151121, doi:10.1128/jvi.01511-21 (2021).

20 Rathnasinghe, R. et al. The N501Y mutation in SARS-CoV-2 spike leads to morbidity in obese and aged mice and is neutralized by convalescent and post-vaccination human sera. medRxiv : the preprint server for health sciences, doi:10.1101/2021.01.19.21249592 (2021).

$21 \mathrm{Gu}, \mathrm{H}$. et al. Adaptation of SARS-CoV-2 in BALB/c mice for testing vaccine efficacy. Science 369, 1603-1607, doi:10.1126/science.abc4730 (2020).

22 Chen, R. E. et al. In vivo monoclonal antibody efficacy against SARS-CoV-2 variant strains. Nature, doi:10.1038/s41586-021-03720-y (2021). 
23 Kibler, K. V. et al. Intranasal immunization with a vaccinia virus vaccine vector expressing prefusion stabilized SARS-CoV-2 spike fully protected mice against lethal challenge with the heavily mutated mouse-adapted SARS2-N501Y<sub>MA30</sub> strain of SARS-CoV-2. bioRxiv, 2021.2012.2006.471483, doi:10.1101/2021.12.06.471483 (2021).

24 Leist, S. R. et al. A Mouse-Adapted SARS-CoV-2 Induces Acute Lung Injury and Mortality in Standard Laboratory Mice. Cel/ 183, 1070-1085.e1012, doi:10.1016/j.cell.2020.09.050 (2020).

25 Dinnon, K. H., 3rd et al. A mouse-adapted model of SARS-CoV-2 to test COVID-19 countermeasures. Nature 586, 560-566, doi:10.1038/s41586-020-2708-8 (2020).

26 Roy Wong, L.-Y. et al. Eicosanoid signaling as a therapeutic target in middle-aged mice with severe COVID-19. bioRxiv, 2021.2004.2020.440676, doi:10.1101/2021.04.20.440676 (2021).

27 Vanderheiden, A. et al. CCR2 Signaling Restricts SARS-CoV-2 Infection. mBio 12, e0274921, doi:10.1128/mBio.02749-21 (2021).

28 Muruato, A. et al. Mouse-adapted SARS-CoV-2 protects animals from lethal SARS-CoV challenge. PLoS Bio/ 19, e3001284, doi:10.1371/journal.pbio.3001284 (2021).

29 Kuiper, M. J. et al. <em>But Mouse, you are not alone</em>: On some severe acute respiratory syndrome coronavirus 2 variants infecting mice. bioRxiv, 2021.2008.2004.455042, doi:10.1101/2021.08.04.455042 (2021).

30 Wei, C. et al. Evidence for a mouse origin of the SARS-CoV-2 Omicron variant. bioRxiv, 2021.2012.2014.472632, doi:10.1101/2021.12.14.472632 (2021).

31 Cameroni, E. et al. Broadly neutralizing antibodies overcome SARS-CoV-2 Omicron antigenic shift. bioRxiv, 2021.2012.2012.472269, doi:10.1101/2021.12.12.472269 (2021).

32 Sia, S. F. et al. Pathogenesis and transmission of SARS-CoV-2 in golden hamsters. Nature 583, 834-838, doi:10.1038/s41586-020-2342-5 (2020).

33 Imai, M. et al. Syrian hamsters as a small animal model for SARS-CoV-2 infection and countermeasure development. Proc Natl Acad Sci U S A 117, 16587-16595, doi:10.1073/pnas.2009799117 (2020).

34 Ying, B. et al. Protective activity of mRNA vaccines against ancestral and variant SARS-CoV-2 strains. Sci Transl Med, eabm3302 (2021).

35 Imai, M. et al. Characterization of a new SARS-CoV-2 variant that emerged in Brazil. Proc Natl Acad Sci U S A 118, doi:10.1073/pnas.2106535118 (2021). 
36 Winkler, E. S. et al. Human neutralizing antibodies against SARS-CoV-2 require intact Fc effector functions for optimal therapeutic protection. Cell 184, 1804-1820.e1816, doi:10.1016/j.cell.2021.02.026 (2021).

37 Simpson, S. et al. Radiological Society of North America Expert Consensus Statement on Reporting Chest CT Findings Related to COVID-19. Endorsed by the Society of Thoracic Radiology, the American College of Radiology, and RSNA - Secondary Publication. Journal of thoracic imaging 35, 219227, doi:10.1097/rti.0000000000000524 (2020).

38 Damas, J. et al. Broad host range of SARS-CoV-2 predicted by comparative and structural analysis of ACE2 in vertebrates. Proc Natl Acad Sci U S A 117, 22311-22322, doi:10.1073/pnas.2010146117 (2020).

39 Gilliland, T. et al. Protection of human ACE2 transgenic Syrian hamsters from SARS CoV-2 variants by human polyclonal IgG from hyper-immunized transchromosomic bovines. bioRxiv, doi:10.1101/2021.07.26.453840 (2021).

40 Espenhain, L. et al. Epidemiological characterisation of the first 785 SARS-CoV-2 Omicron variant cases in Denmark, December 2021. Euro Surveil/ 26, doi:10.2807/1560-7917.es.2021.26.50.2101146 (2021).

41 Kupferschmidt, K. \& Vogel, G. How bad is Omicron? Some clues are emerging. Science 374, 13041305, doi:10.1126/science.acx9782 (2021).

42 Michael, C. W. C. et al. Nature Portfolio, doi:10.21203/rs.3.rs-1189219/v1 (2021).

43 Yadav, P. et al. Isolation of SARS-CoV-2 B.1.1.28.2 (P2) variant and pathogenicity comparison with D614G variant in hamster model. Journal of infection and public health 15, 164-171, doi:10.1016/j.jiph.2021.12.009 (2021).

44 Ulrich, L. et al. Enhanced fitness of SARS-CoV-2 variant of concern Alpha but not Beta. Nature, doi:10.1038/s41586-021-04342-0 (2021).

45 Abdelnabi, R. et al. The omicron (B.1.1.529) SARS-CoV-2 variant of concern does not readily infect Syrian hamsters. bioRxiv, 2021.2012.2024.474086, doi:10.1101/2021.12.24.474086 (2021).

46 Zheng, J. et al. COVID-19 treatments and pathogenesis including anosmia in K18-hACE2 mice. Nature 589, 603-607, doi:10.1038/s41586-020-2943-z (2021).

47 Mannar, D. et al. SARS-CoV-2 Omicron Variant: ACE2 Binding, Cryo-EM Structure of Spike ProteinACE2 Complex and Antibody Evasion. bioRxiv, 2021.2012.2019.473380, doi:10.1101/2021.12.19.473380 (2021). 
48 Zang, R. et al. TMPRSS2 and TMPRSS4 promote SARS-CoV-2 infection of human small intestinal enterocytes. Sci Immuno/ 5, doi:10.1126/sciimmunol.abc3582 (2020).

49 Matsuyama, S. et al. Enhanced isolation of SARS-CoV-2 by TMPRSS2-expressing cells. Proc Natl Acad Sci U S A 117, 7001-7003, doi:10.1073/pnas.2002589117 (2020).

50 Chen, R. E. et al. Resistance of SARS-CoV-2 variants to neutralization by monoclonal and serumderived polyclonal antibodies. Nat Med, doi:10.1038/s41591-021-01294-w (2021).

51 Plante, J. A. et al. Spike mutation D614G alters SARS-CoV-2 fitness. Nature, doi:10.1038/s41586020-2895-3 (2020).

52 Gagne, M. et al. Protection from SARS-CoV-2 Delta one year after mRNA-1273 vaccination in rhesus macaques coincides with anamnestic antibody response in the lung. Cell, doi:10.1016/j.cell.2021.12.002 (2021).

53 Corbett, K. S. et al. mRNA-1273 protects against SARS-CoV-2 beta infection in nonhuman primates. Nat Immunol, doi:10.1038/s41590-021-01021-0 (2021).

$54 \mathrm{Li}$, Z. et al. Generation of transgenic pigs by cytoplasmic injection of piggyBac transposase-based pmGENIE-3 plasmids. Biology of reproduction 90, 93, doi:10.1095/biolreprod.113.116905 (2014).

55 Case, J. B., Bailey, A. L., Kim, A. S., Chen, R. E. \& Diamond, M. S. Growth, detection, quantification, and inactivation of SARS-CoV-2. Virology 548, 39-48, doi:10.1016/j.virol.2020.05.015 (2020).

56 Vanderheiden, A. et al. CCR2-dependent monocyte-derived cells restrict SARS-CoV-2 infection. bioRxiv, doi:10.1101/2021.05.03.442538 (2021).

57 Chu, D. K. W. et al. Molecular Diagnosis of a Novel Coronavirus (2019-nCoV) Causing an Outbreak of Pneumonia. Clin Chem, doi:10.1093/clinchem/hvaa029 (2020).

58 Halfmann, P. et al. SARS-CoV-2 Interference of Influenza Virus Replication in Syrian Hamsters. $J$ Infect Dis, doi:10.1093/infdis/jiab587 (2021).

59 Chung, M. et al. CT Imaging Features of 2019 Novel Coronavirus (2019-nCoV). Radiology 295, 202-207, doi:10.1148/radiol.2020200230 (2020).

\section{Figures}


Body weight 16-17 wk old 129 mice (MD)

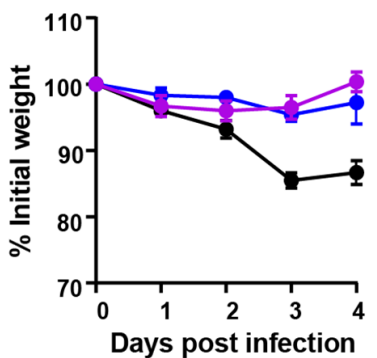

b

Viral RNA load (4 dpi) 129 mice (MD)
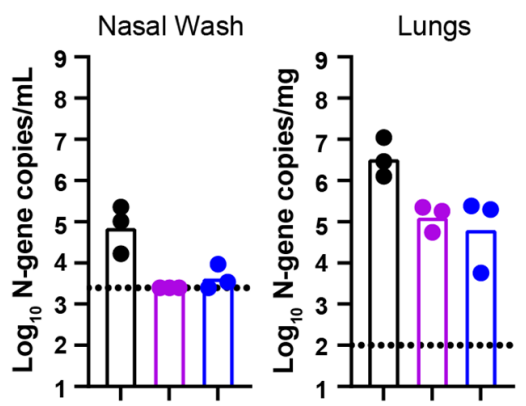

e
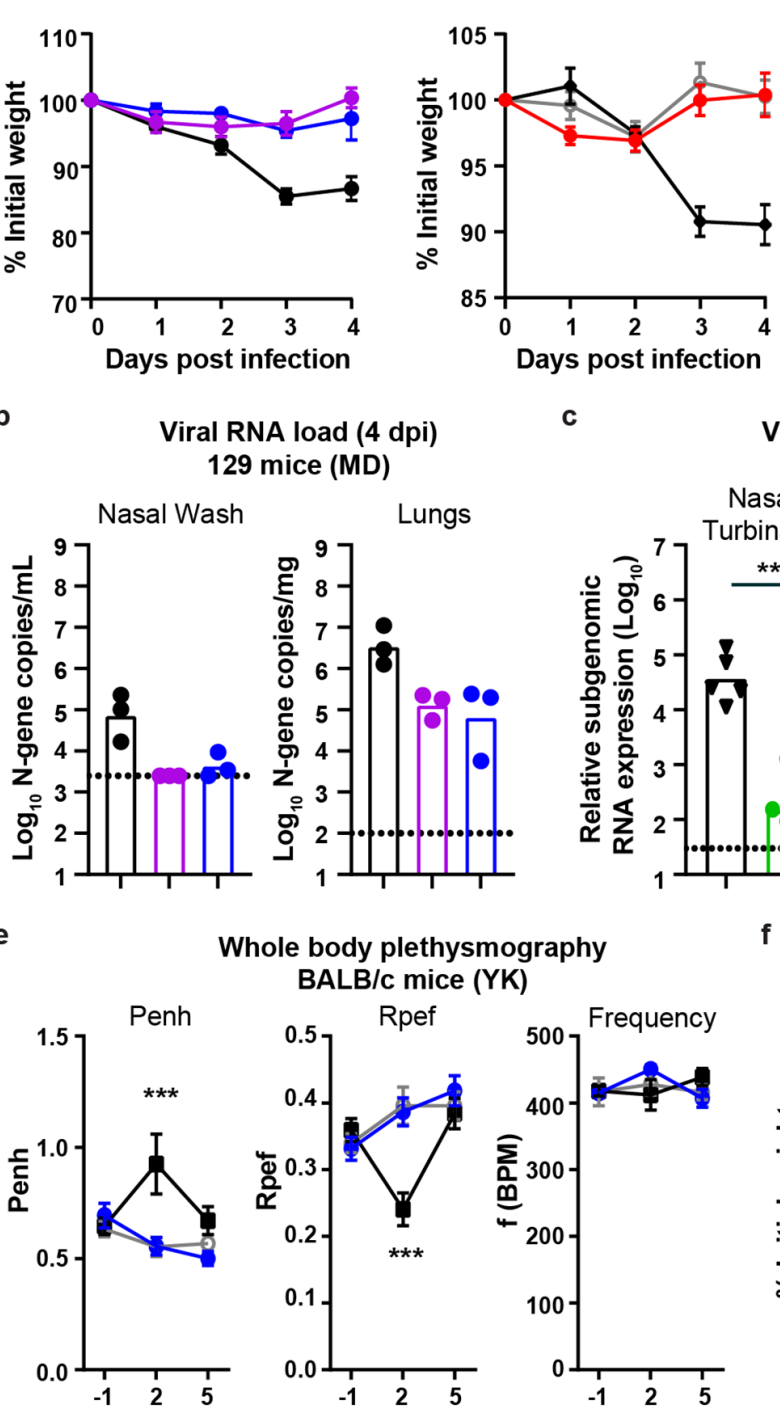

c
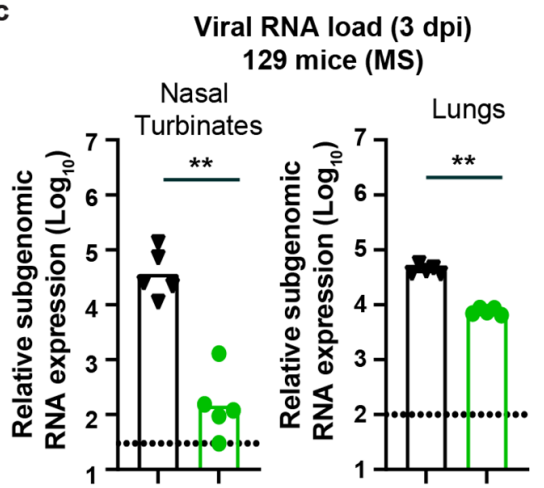

f Body weight 5 mo old K18-hACE2 mice (MD) 6 mo old K18-hACE2 mice (AGS)

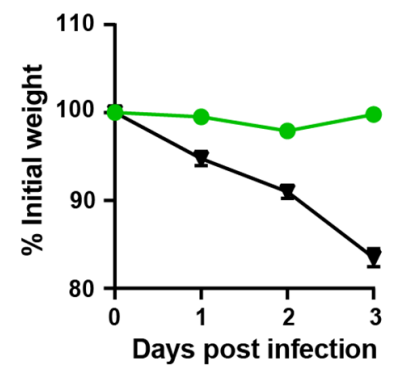

d

d Infectious virus titer (2 dpi)

BALB/c mice (YK)

Nasal
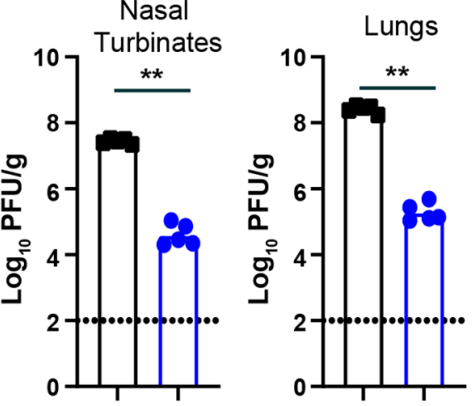

g

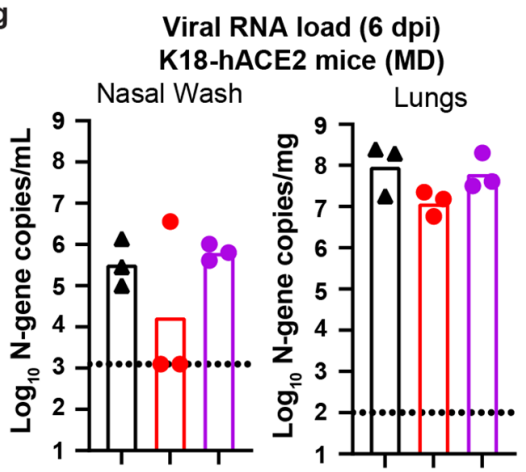

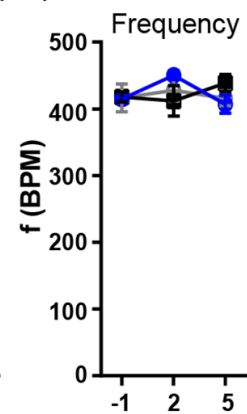

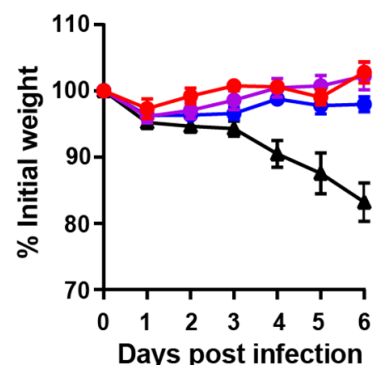

- Mock

- $10^{5}$ FFU N501Y/D614G

- $10^{3}$ FFU D614G

- $10^{3}$ PFU B. 1.351

- $10^{4}$ PFU B.1.351

- $10^{5}$ PFU B. 1.351

> $10^{6}$ PFU B. 1.351

$\star 10^{5}$ PFU B.1.1.7

- $10^{3} \mathrm{FFU}$ or PFU B.1.1.529

- $10^{4}$ FFU B.1.1.529

- $10^{5}$ FFU or PFU B.1.1.529

- $10^{6}$ PFU B.1.1.529

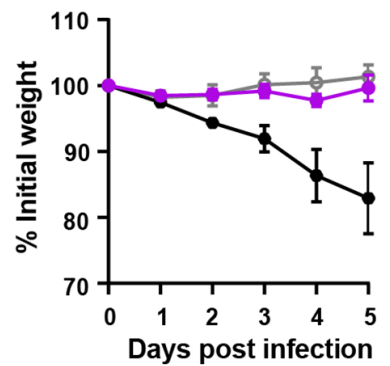

Figure 1

\section{Figure 1}

B.1.1.529 is attenuated in different mouse models of SARS-CoV-2 infection. a, Far left. Weight change in 129 mice inoculated via intranasal route with $10^{4}$ (purple circles, $n=6$ ) or $10^{5}$ (blue circles, $n=6$ ) FFU of B.1.1.529 (strain hCoV-19/USA/WI-WSLH-221686/2021) or $10^{5}$ FFU of WA1/2020 N501Y/D614G (black circles, $n=6$ ). Center left. Weight change in mock-infected 129 mice (grey circles, $n=4$ ) or 129 mice inoculated intranasally with $10^{3}$ (red circles, $n=5$ ) PFU of B.1.1.529 (strain hCoV-19/USA/NY-MSHSPSP- 
PV44476/2021) or B.1.351 variant of SARS-CoV-2 (black diamonds, $\mathrm{n}=3$ ). Center right. Weight change in 129 mice inoculated intranasally with $10^{6}$ PFU of B.1.1.529 (strain hCoV19/EHC_C19_2811C, green circles, $n=5$ ) or B.1.351 (black triangles, $n=5$ ). Far right. Weight change in 10 to 14-month-old C57BL/ 6 mice inoculated intranasally with $10^{5}$ PFU of B.1.1.529 (strain hCoV19/EHC_C19_2811C, blue circles, $n=$ 4), B.1.1.7 (black star, $n=10$ ), or B.1.351 (black squares, $n=18$ ). Data are mean \pm SEM. b, Nasal wash and lung viral RNA levels in 129 mice inoculated with $10^{4}$ (purple circles, $n=3$ ) or $10^{5}$ (blue circles, $n=3$ ) FFU of B.1.1.529 (strain hCoV-19/USA/WI-WSLH-221686/2021) or 105 FFU of WA1/2020 N501Y/D614G (black circles, $n=3$ ). c, Nasal turbinates and lung viral RNA levels in 129 mice inoculated with $10^{6}$ PFU of B.1.1.529 (strain hCoV19/EHC_C19_2811C, green circles, $n=5)$ or B.1.351 (black triangles, $n=5)(* * ~ P<$ 0.01 , by Mann-Whitney $U$ test). $d$, Nasal turbinates and lung virus titers from BALB/c mice inoculated with 105 PFU of B.1.1.529 (strain hCoV-19/Japan/NC928-2N/2021, blue circles, $n=5$ ) or B.1.351 (black squares, $\mathrm{n}=5$ ) (** $P<0.001$, by Mann-Whitney $U$ test). e, Pulmonary function analysis in infected BALB/C mice. Penh, a surrogate marker for bronchoconstriction or airway obstruction, was measured by whole body plethysmography. Data are presented as the mean \pm SEM. $P$ values were calculated by using pairwise comparisons after a linear mixed model analysis $(* * * P<0.001)$. Asterisks indicate statistically significant differences between B.1.351-infected $(n=5)$ and B.1.1.529-infected $(n=5)$ or uninfected animals $(n=5) . f$, Left: Weight change in 5-month-old K18-hACE2 transgenic mice inoculated intranasally with $10^{3}$ (red circles, $n=3$ ), $10^{4}$ (purple circles, $n=6$ ), or $10^{5}$ (blue circles, $n=3$ ) FFU of B.1.1.529 (strain hCoV-19/USA/WI-WSLH-221686/2021) or $10^{3}$ FFU of WA1/2020 D614G (black triangles, $\mathrm{n}=6$ ). Right: Weight change in 6-month-old K18-hACE2 transgenic mice inoculated intranasally with $10^{4}$ (purple circles, $n=6$ ) PFU of B.1.1.529 (strain hCoV-19/USA/NY-MSHSPSP-PV44476/2021), or $10^{4}$ PFU of B.1.351 (black hexagon, $n=6$ )). Age-matched uninfected mice (grey circles, $n=4$ ) were included as controls. Data are presented as the mean \pm SEM. g, Nasal wash and lung viral RNA levels in K18-hACE2 mice inoculated with $10^{3} \mathrm{FFU}$ of WA1/2020 D614G (black triangles, $n=3$ ), or $10^{3}$ (red circles, $n=3$ ) or $10^{4}$ (purple circles, $n=3$ ) FFU of B.1.1.529 (strain hCoV-19/USA/WI-WSLH-221686/2021). The results are from one experiment and each symbol in $\mathbf{b}, \mathbf{c}, \mathbf{d}$, and $\mathbf{g}$ represents an individual animal. The dotted line is the limit of detection. Infection studies in panels $\mathbf{a}, \mathbf{b}$ and $\mathbf{f}$ with WA1/2020 N501Y/D614G are shown as comparisons and were adapted from published data 22 . 
Body weight

Syrian hamster (YK)
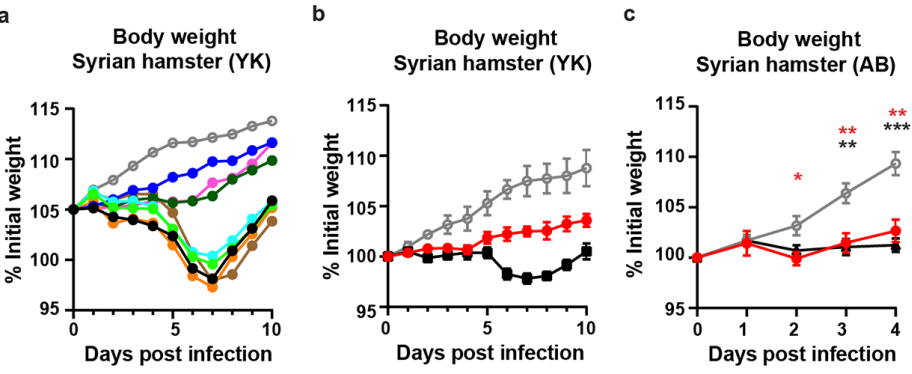

Syrian hamster (RS)
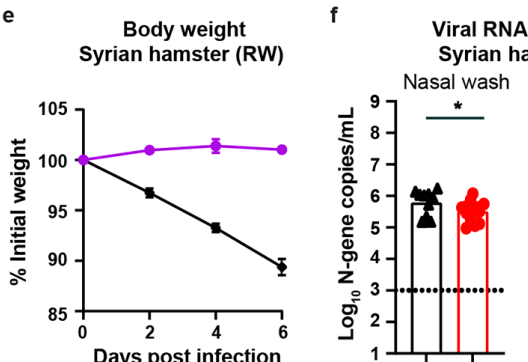

g
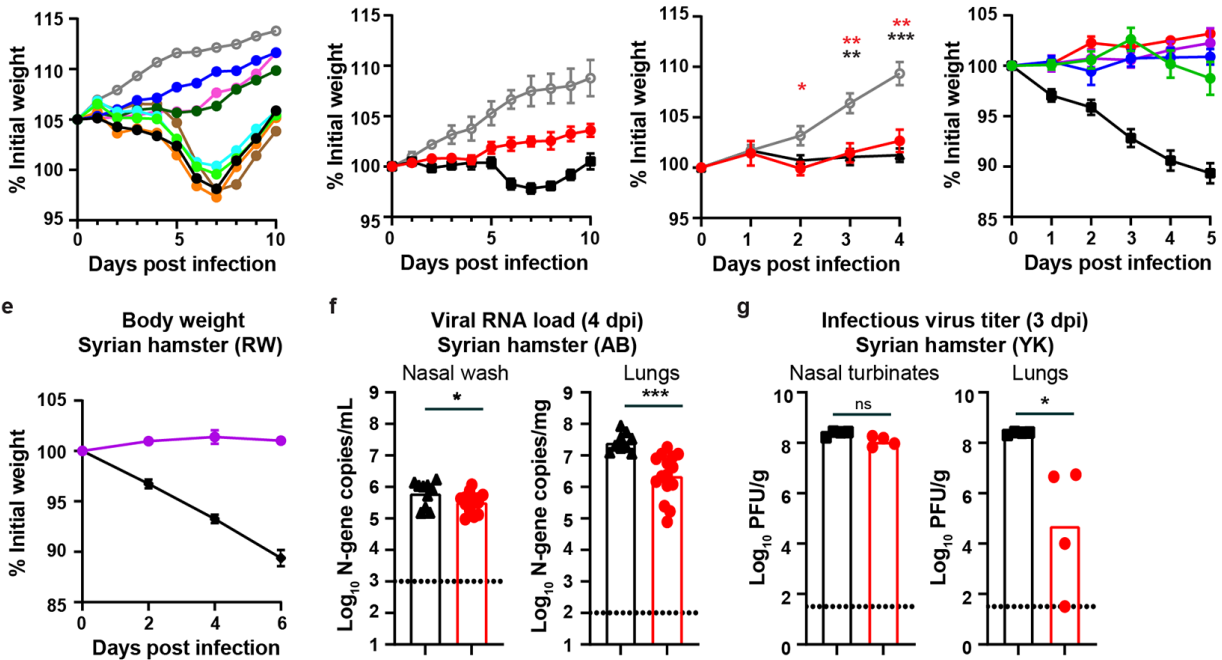

rian hamster (YK)

h Infectious virus titer (4 dpi) Syrian hamster (RW)

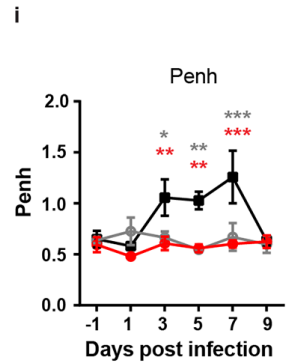

Whole body plethysmography Syrian hamster (YK)
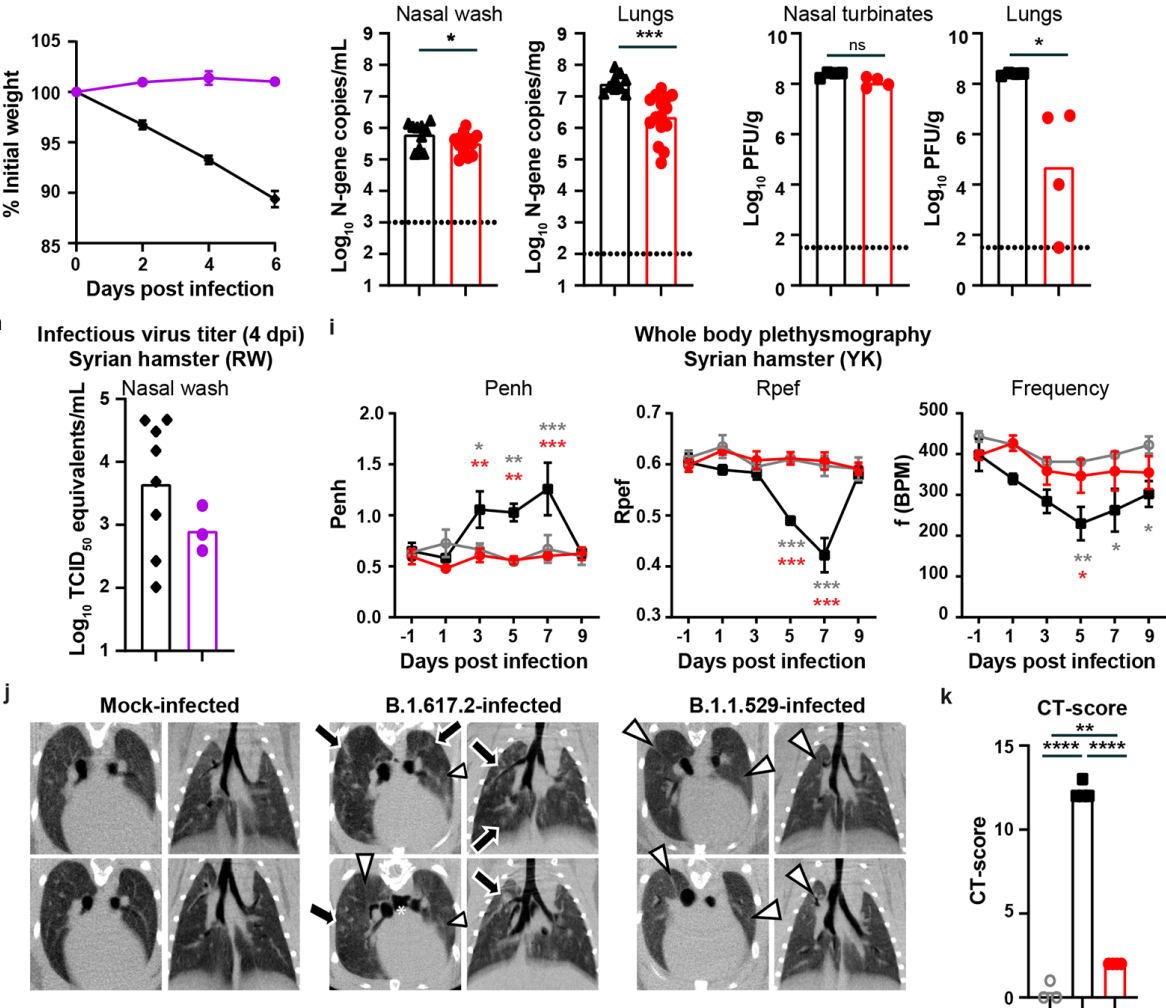
hACE2 hamsters (YK)
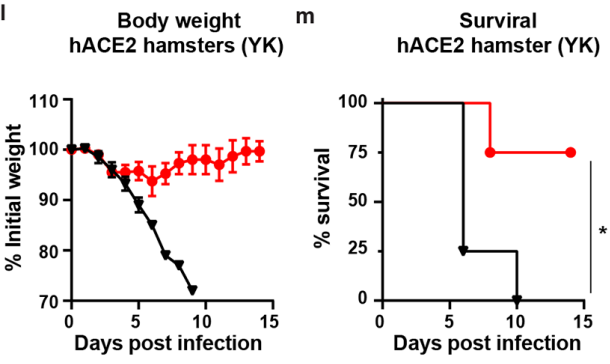

n

Infectious virus titer ( 3 and $\mathbf{5} \mathrm{dpi}$ )

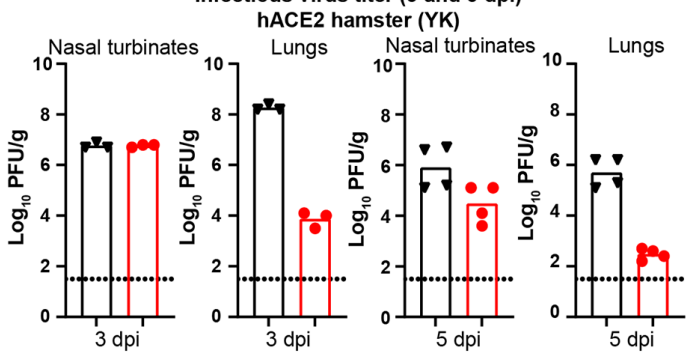

- $10^{5}$ PFU Epsilon (VRLC009)

- Mock

- $10^{5}$ PFU Wuhan (NC002)

- $10^{5}$ PFU Beta (HP01542)

- $10^{5}$ PFU Delta (UW-5250)

$10^{5}$ PFU lota (PV26425)

$10^{5}$ PFU Mu (80384)

- $10^{5}$ PFU Lambda (SEC0506)

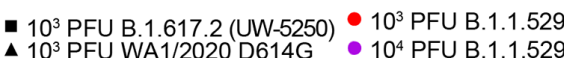

- $10^{3}$ PFU HP-095 D614G

- $10^{4}$ PFU WA1/2020
- $10^{6}$ PFU B.1.1.529

Figure 2

Figure 2

B.1.1.529 is attenuated in wild-type and human ACE2-transgenic Syrian hamsters. a, Weight change in uninfected age-matched Syrian hamsters (open grey circles, $n=4$ ) or in hamsters inoculated intranasally with $10^{5} \mathrm{PFU}(\mathrm{n}=9)$ of B.1.1.529 (strain hCoV-19/Japan/NC928-2N/2021, blue circles), B.1.351 (Beta, HP01542), B.1.617.2 (Delta, strain hCoV-19/USA/WI-UW-5250/2021), B.1.621 (Mu, 80384), B.1.427 (Epsilon, VRLC009), B.1.526 (lota, PV26425), C.37 (Lambda, SEC0506), or Wuhan-1 (black circles). b, 
Weight change in uninfected age-matched Syrian hamsters (open grey circles, $n=3$ ) or in hamsters inoculated intranasally with $10^{3}$ PFU of B.1.1.529 (strain hCoV-19/Japan/NC928-2N/2021, red circles, $n=$ 4) or B.1.617.2 (black squares, $n=4$ ). Data are mean \pm SEM. c, Weight change in uninfected age-matched Syrian hamsters (open grey circles, $n=9$ ) or in hamsters inoculated intranasally with $10^{3} \mathrm{PFU}$ of B.1.1.529 (strain hCoV-19/USA/WI-WSLH-221686/2021, red circles, $\mathrm{n}=10$ ) or WA1/2020 D614G (black triangles, $\mathrm{n}=6)$. Data are mean \pm SEM. ( $P<0.05$, $* * P<0.01$, $* \star * ~ P<0.001$ by two-way ANOVA with a Dunnett's correction). d, Weight change in Syrian hamsters inoculated intranasally with $10^{3}$ (red circles, $n$ $=4$ ), $10^{4}$ (purple circles, $n=4$ ), $10^{5}$ (blue circles, $n=4$ ), or $10^{6}$ (green circles, $n=4$ ) PFU of B.1.1.529 (strain hCoV19/EHC_C19_2811C) or $10^{3}$ PFU of B.1.617.2 (black squares, $n=4$ ). Data are mean \pm SEM. $\mathbf{e}$, Weight change in hamsters inoculated intranasally with $10^{4}$ PFU of B.1.1.529 (strain hCoV19/EHC_C19_2811C, purple circles, $n=5$ ) or WA1/2020 (black diamonds, $n=9$ ). Data are mean \pm SEM. f, Nasal wash and lung viral RNA load in wild-type Syrian hamsters inoculated with $10^{3} \mathrm{PFU}$ of WA1/2020 D614G (black triangles, $n=15$ ) or B.1.1.529 (strain hCoV-19/USA/WI-WSLH-221686/2021, red circles, $\mathrm{n}=15$ ) (*** $P<0.001, * P<0.05$ by unpaired t-test). $\mathbf{g}$, Nasal turbinates and lung virus titer in wildtype Syrian hamsters inoculated with $10^{3}$ PFU of B.1.617.2 (strain hCoV-19/USA/WI-UW-5250/2021, black squares, $n=4$ ) or B.1.1.529 (strain hCoV-19/Japan/NC928-2N/2021, red circles, $n=4)(* P<0.05$, $\mathrm{ns}=$ not significant; Mann Whitney $U$ test). $h$, Nasal wash viral RNA load (TCID ${ }_{50}$ equivalents $\left./ \mathrm{mL}\right)$ in wildtype Syrian hamsters inoculated with $10^{4}$ PFU of WA1/2020 (black diamonds, $n=8$ ) or B.1.1.529 (strain hCoV19/EHC_C19_2811C, purple circles, $n=3$ ). i, Pulmonary function analysis in infected Syrian hamsters. Penh was measured by whole body plethysmography. Data are mean \pm SEM. $P$ values were calculated by using pairwise comparisons after a linear mixed model analysis ( $* * * P<0.001$, $* \star P<0.01$, * $P<0.05)$. Asterisks indicate statistically significant differences between B.1.617.2-infected $(\mathrm{n}=4)$ and B.1.1.529-infected $(n=4)$ or uninfected animals $(n=3)$. j, Representative micro-CT axial and coronal images of the lungs of mock-infected $(n=3)$ or B.1.617.2- $(n=4)$ and B.1.1.529-infected $(n=4)$ hamsters on $7 \mathrm{dpi}$. Lung abnormalities included multifocal nodules (black arrows), ground glass opacity (white arrowheads), and regions of lung consolidation (white arrows) that were peripheral, bilateral, and multilobar. Pneumomediastinum is indicated with white asterisks. $\mathbf{k}$, CT score for uninfected hamsters (open grey circles, $n=3$ ) or those inoculated with $10^{3}$ PFU of B.1.617.2 (black squares, $n=4$ ) or B.1.1.529 (strain hCoV-19/Japan/NC928-2N/2021, red circles, n = 4). I, Weight change in hACE2-transgenic Syrian hamsters inoculated intranasally with $10^{3}$ PFU of HP-095 D614G (black triangles, $n=4$ ) or B.1.1.529 (strain hCoV-19/USA/WI-WSLH-221686/2021, red circles, $n=4$ ). m, Survival analysis of hACE2transgenic Syrian hamsters after inoculation with $10^{3}$ PFU of HP-095 D614G (black triangles, $n=4$ ) or B.1.1.529 (strain hCoV-19/USA/WI-WSLH-221686/2021, red circles, $n=4)(* P<0.05$; log-rank test). $\mathbf{n}$, Nasal turbinate and lung infectious virus titer by plaque assay at 3 and 5 dpi from hACE2-transgenic Syrian hamsters inoculated with $10^{3}$ PFU of HP-095 D614G (black triangles) or B.1.1.529 (strain hCoV19/USA/WI-WSLH-221686/2021, red circles); $n=3$ (3 dpi), $n=4$ (5 dpi). The results in the Figure are from one (a, b, d-e, and $\mathbf{g}-\mathbf{n})$ or two to three independent (c, and $\mathbf{f})$ experiments. Each symbol represents an individual animal. Dotted lines represent the limit of detection. 
a

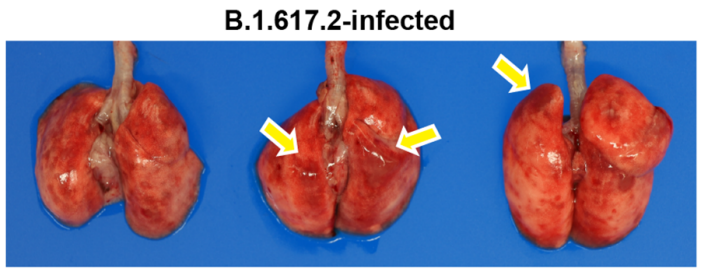

b

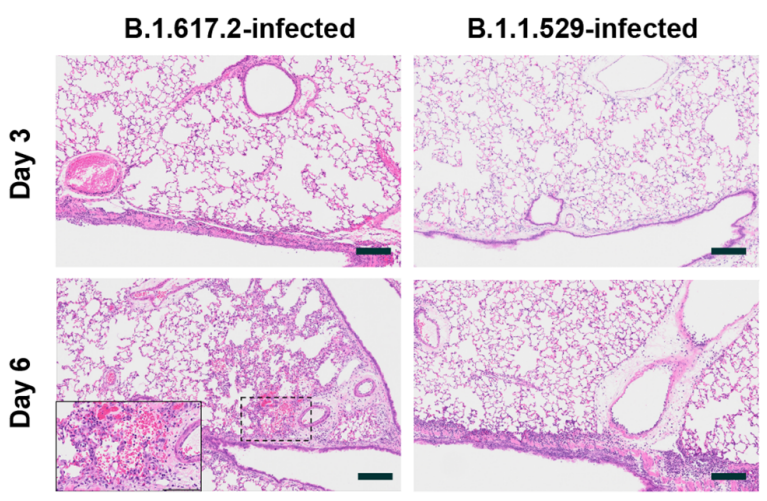

d
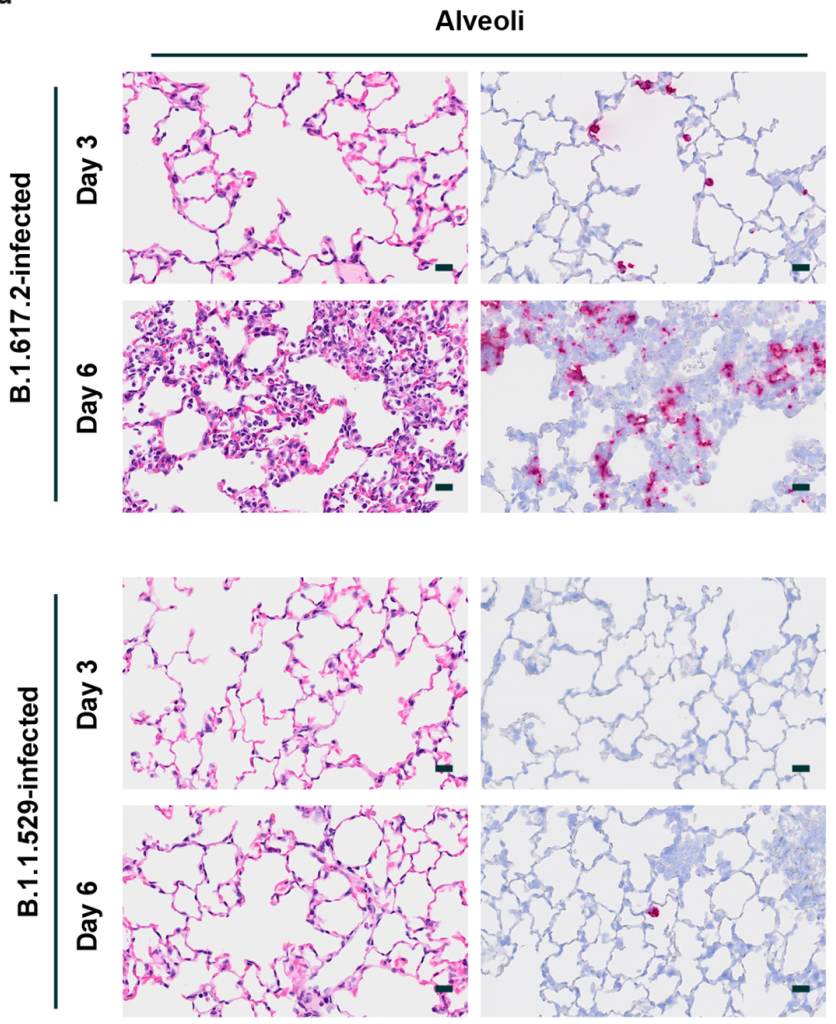

B.1.1.529-infected

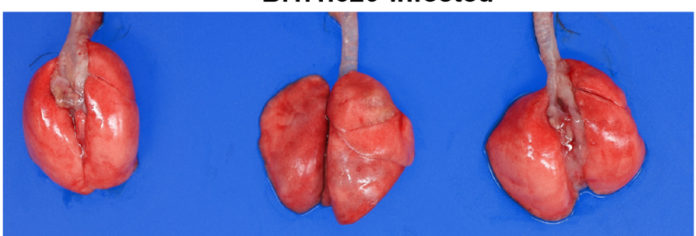

c

Histology score

Syrian hamster

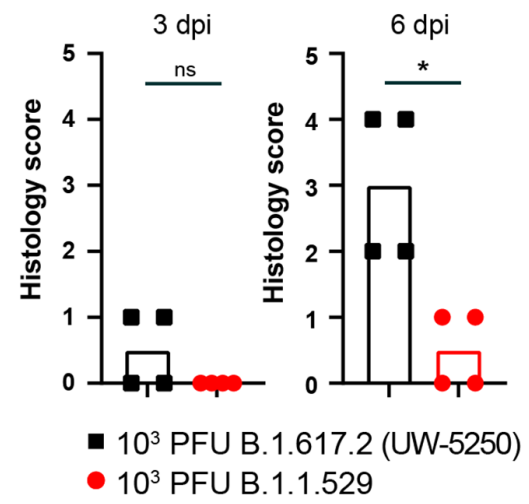

Bronchus
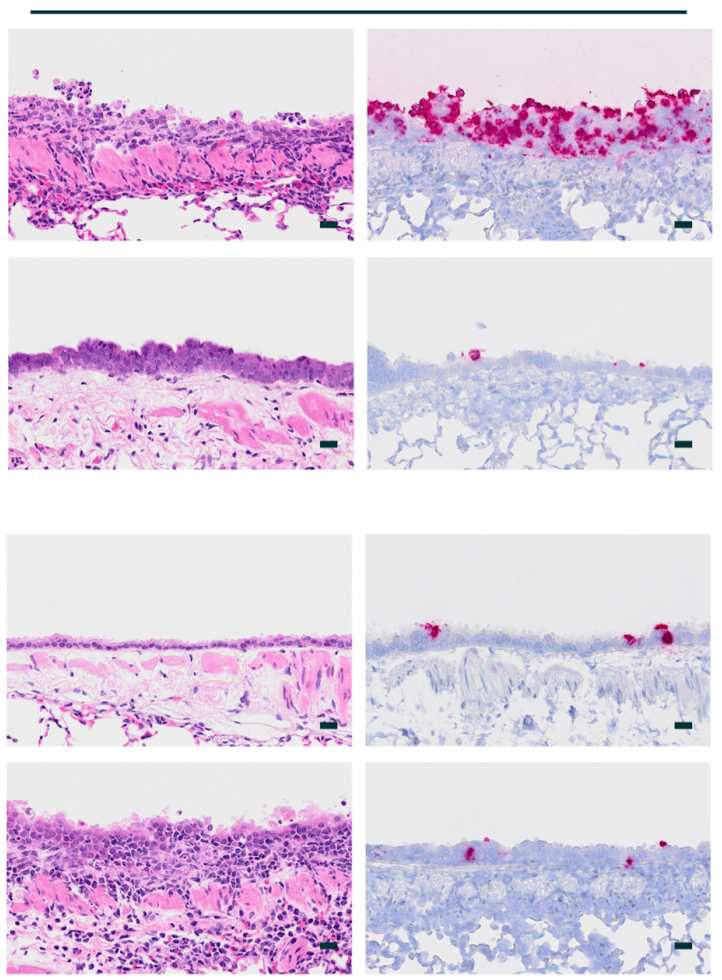

Figure 3

\section{Figure 3}

Pathological findings in the lungs of SARS-CoV-2 infected Syrian hamsters.

The hamsters were infected with B.1.617.2 or B.1.1.529 variant of SARS-CoV-2 and sacrificed on 3 days $(n=4)$ and 6 days $(n=4)$ post infection for histopathological examinations. a, Macroscopic images of the lungs obtained from B.1.617.2 or B.1.1.529-infected Syrian hamsters at 6 dpi. Yellow arrows indicate 
areas of pulmonary hemorrhage. b, Representative histopathological images for the lung sections obtained from the animals infected with B.1.617.2 or B.1.1.529 at 3 or $6 \mathrm{dpi}$ are shown at low magnification. Scale bars, $200 \mu \mathrm{m}$. Focal alveolar hemorrhage found in B.1.617.2-infected animals at 6 dpi is highlighted by dashed lines and shown at higher magnification in the inset image (scale bar indicating $100 \mu \mathrm{m}$ ). c, Histopathological score of viral pneumonia in the lungs of infected hamsters. Score was determined based on the percentage of alveolitis in a given section collected from each animal in each group using the following scoring: 0 , no pathological change; 1 , affected area ( $\leq 10 \%) ; 2$, affected area $(<50 \%,>10 \%) ; 3$, affected area $(\geq 50 \%)$; an additional point was added when pulmonary edema and/or alveolar hemorrhage was observed. Data are expressed as scatter plots with the median score \pm $95 \%$ confidential interval. Each dot represents the score of each animal. Comparison between B.1.617.2 $(n=4)$ or B.1.1.529 $(n=4)$ was performed using Kruskal-Wallis test with a Dunn's post-tests. ns, not significant; *, $P<0.05$. d, RNA in situ hybridization (RNA-ISH) for SARS-CoV-2 viral RNA. Representative RNA-ISH images for the alveoli and bronchi of hamsters infected with B.1.617.2 $(n=4)$ or B.1.1.529 $(n=$ 4) virus at 3 or $6 \mathrm{dpi}$ are shown. Left panels, alveolar region. Right panels, bronchial region. Scale bars, 20 $\mu \mathrm{m}$. 